On associe en général la musique à la beauté et à l'authenticité. La musique invite à la contemplation, à la détente, au plaisir de l'écoute et à l'épanouissement de jouer d'un instrument. Musique et stress, ou musique et douleur, ne sont des liens reconnaissables qu'au deuxième abord et ils étaient (et sont parfois encore) des sujets tabous pour toutes les personnes concernées. La médecine des musiciens est une toute nouvelle spécialité. Ce n'est que depuis les années 1980 qu'elle a été considérée sous l'angle de la «médecine du travail». Qu’y a-t-il de particulier dans cette profession et pourquoi est-il important de s'occuper spécialement des musiciens? Cet article a pour but de répondre à ces questions et de montrer comment les efforts actuels dans ce domaine sont mis en œuvre dans notre pays.

Dr Carlos Beat Quinto,

\title{
La médecine des musiciens: discipline insolite ou nécessité?
}

\author{
Martina Berchtold-Neumann \\ Présidente de la SMM, psychologue diplômée FSP
}

Durant leur vie professionnelle, souvent même au cours de leur formation, de nombreux musiciens développent des troubles physiques et psychiques caractéristiques, qui sont au moins en partie causés par la pratique professionnelle d'un instrument ou du chant. Les syndromes douloureux sont en tête de liste des plaintes chez les musiciens. Il n'est pas difficile de s'imaginer une violoniste qui doit se tordre l'avantbras gauche pendant des heures tout en bougeant son archet du bras droit afin de pouvoir enseigner la musique ou jouer dans un orchestre philarmonique. La santé des joueurs d'instruments à vent est également très sollicitée, qu'il s'agisse de postures pénibles ou de la pression intense exercée sur les lèvres et les dents. Ou encore les douleurs du système musculosquelettique qui découlent principalement d'un travail permanent et intensif avec un «outil de travail» non ergonomique, pour la simple raison que les instruments ne sont généralement pas fabriqués pour correspondre à nos aptitudes physiques naturelles et, pour en jouer, il faut souvent adopter des postures à la limite de la physiologie. Les dispositions physique et psychique des musiciens, leur environnement professionnel et privé, le répertoire, les heures de répétition et la technique instrumentale personnelle influent aussi sur le développement de troubles tout comme les risques de blessure ou de maladie peuvent rapidement devenir un obstacle insurmontable pour un musicien professionnel. Enfin, les pathologies liées au système auditif et à l'oreille sont de plus en plus cou- rantes en raison du volume toujours plus élevé des représentations.

Les problèmes relèvent assez souvent du domaine psychique, en particulier du stress. Le trac joue ici un rôle particulièrement important. Là aussi, on peut facilement imaginer ce que cela signifie de se hisser plusieurs soirs par semaine à son meilleur niveau, à $19 \mathrm{~h} 30$ précises, dans les plus grandes salles de Suisse comme la Tonhalle de Zurich. Les artistes sont des perfectionnistes, le public est audiophile et le monde de la musique aime la performance; aucune erreur n'est donc pardonnée. Les futurs musiciens professionnels gran-

Durant leur vie professionnelle, souvent même au cours de leur formation, de nombreux musiciens développent des troubles physiques et psychiques caractéristiques.

dissent dans une culture de la perfection et, même sur un plan intrapsychique, ils ne sont que difficilement en mesure de se pardonner une erreur. Ce sont là les principales origines du trac et de tous ses effets physiologiques. Il n'est pas rare non plus que des horaires de travail irréguliers, les structures hiérarchiques dans les orchestres, la concurrence entre musiciens et souvent aussi des problèmes financiers mènent, en cas de prédisposition et de ressources individuelles insuffisantes, à un problème d'épuisement professionnel. Les maladies des musiciens se caractérisent donc par de nombreux facteurs, qui diffèrent selon l'individu, 
et sont accompagnées de symptômes extrêmement variés se retrouvant rarement sous cette forme et avec les mêmes conséquences dans d'autres groupes professionnels. Par conséquent, il faut souvent une prise en charge spécifique par des médecins et des thérapeutes ayant une connaissance approfondie de la profession et des particularités du jeu instrumental et du chant au niveau professionnel.

La Société suisse pour la médecine de la musique (SMM) a été fondée à Berne en 1997 dans le but de promouvoir la médecine des musiciens. Le point de départ de la SMM a été un problème de santé de l'ancienne tromboniste et initiatrice Pia Bucher, qui cherchait alors une solution et avait du mal à trouver en Suisse des interlocuteurs appropriés. Aujourd'hui encore, cette approche pratique est d'un intérêt central dans le travail de la SMM. Nous voulons aider les musiciens dans l'exercice de leur profession et les soutenir dans leur travail quotidien. C'est dans ce but que nous avons créé notre propre centre de conseil gratuit, actuelle-

\section{Nous voulons aider les musiciens dans l'exer- cice de leur profession et les soutenir dans leur travail quotidien.}

ment dirigé par notre vice-président, le Dr Peter Schönenberger. Les personnes concernées peuvent y faire part de leurs préoccupations, tout comme les «musiciens amateurs», qui sont nombreux en Suisse à jouer dans des ensembles non professionnels. Les musiciens peuvent être orientés vers des médecins ou des thérapeutes compétents qui sont, dans les meilleurs cas, proches de leur domicile ou de leur lieu de travail. Des consultations interdisciplinaires pour musiciens sont par ailleurs proposées à Bâle, Berne, Locarno, Lucerne et Zurich. Là aussi, les musiciens soucieux de leur santé peuvent s'adresser à des spécialistes compétents. Soulignons aussi les consultations de la Haute école d'art de Zurich, étroitement liées aux offres de physiologie musicale pour l'enseignement, la recherche et le conseil, que le professeur Horst Hildebrandt développe depuis plus de 20 ans. En 2009, elle a notamment repris, élargi et modernisé le laboratoire des mains qui avait été développé en 1964 à l'Institut Max-Planck de Dortmund. Ce laboratoire, qui est unique, aide à justifier scientifiquement une technique instrumentale, un diagnostic et une thérapie adaptés aux conditions physiologiques individuelles.

En plus de l'explication scientifique de la pratique musicale, le principal objectif de la SMM est de mettre en place un réseau de spécialistes interdisciplinaires au niveau suisse susceptible d'être contacté lors de problèmes spécifiques aux musiciens. A l'heure actuelle, nos membres se composent de musiciens, de médecins et psychologues et de thérapeutes de diverses disciplines (un tiers chacun). Nous sommes constamment à la recherche de nouveaux membres afin de pouvoir conseiller encore mieux nos musiciens et, surtout, de les prendre en charge plus près de chez eux.

La coopération avec les associations professionnelles suisses et les sociétés spécialisées étrangères est également importante pour nous. Grâce à une étroite collaboration, nous sommes désormais en mesure d'échanger nos connaissances dans le cadre de congrès communs. Il existe aujourd'hui un grand nombre de publications nationales et internationales sur la médecine des musiciens, tant dans des livres que des revues. La prévention des maladies des musiciens est un axe essentiel de notre travail. Par des conférences, des séminaires et des symposiums, nous essayons de convaincre les futurs musiciens qu'il leur appartient (aussi) de prendre soin d'eux-mêmes.

Le souhait de telles mesures préventives ne provient pas que des professions médicales, mais aussi d'étudiants, de musiciens d'orchestre, de pédagogues instrumentaux et vocaux et d'associations professionnelles. Beaucoup de musiciens ne se sentent pas suffisamment préparés lors de leurs études pour faire face au stress de leur vie professionnelle ultérieure. Dans les orchestres, il manque aussi souvent d'engagement dans le suivi par des médecins du travail ou des physiothérapeutes qui enseignent aux musiciens des mesures générales et spécifiques de prévention et les forment à faire face aux problèmes de santé.

La médecine des musiciens restera importante à l'avenir et continuera aussi de gagner en importance dans le domaine scientifique. Dans un environnement de plus en plus compétitif, dans une société mondialisée où la concurrence et la sélection sont omniprésentes, il est essentiel d'offrir un soutien aux personnes qui peuvent nous apporter beauté, joie et contemplation mais aussi un important ancrage culturel.

\section{$16^{\mathrm{e}}$ symposium de la SMM}

Organisé cette année en collaboration avec la Fondation suisse des artistes interprètes (SIS), le $16^{\mathrm{e}}$ symposium de la SMM sera l'occasion de faire connaître et découvrir notre société et notre travail. II sera consacré au thème de "la douleur: un signal d'alarme» et aura lieu toute la journée du 27 octobre 2018 à Lucerne. Informations et inscriptions sous https://www.musikmedizin.ch/aktuelles-symposium/ ( 5 credits SSMT / Société suisse de médecine du travail) 